Anales de Geografía de la Universidad Complutense ISSN: 0211-9803

http://dx.doi.org/10.5209/rev_AGUC.2016.v36.n1.52713

\title{
La expansión del cultivo de la soja en Campeche, México: Problemática y perspectivas
}

\author{
Flavia Echanove Huacuja ${ }^{1}$ \\ Recibido: 3 de septiembre del 2015 / Enviado a evaluar: 23 de enero del 2016 / Aceptado: 3 de mayo del 2016
}

Resumen. La soja es el grano en el que mayor dependencia alimentaria tiene México, por lo que a partir de 2008 el gobierno ha promovido su cultivo, otorgando relevantes subsidios, tanto a productores como a compradores del grano, contribuyendo así a un reciente proceso de expansión en ciertos estados, como ocurre en Campeche. Este artículo tiene como objetivo el análisis de las características y efectos de dichos apoyos, así como del resto de factores que hasta hoy han llevado a los productores del mencionado estado a iniciar o expandir el cultivo de la soja. Los hallazgos de la investigación revelan que si bien los productores han mejorado sus niveles de ingreso, el proceso es vulnerable, al depender de variables como los apoyos gubernamentales, los precios internacionales de la soja y el tipo de cambio. Aunque el estudio de los efectos negativos de la soja genéticamente manipulada (GM) en otros ámbitos (medio ambiente, biodiversidad, deforestación, salud humana y animal) no es el objetivo de la presente investigación, se proporciona información al respecto, al igual que sobre el conflicto existente entre los productores de dicha soja y los apicultores del estado de Campeche.

Palabras clave: Soja; subsidios; estrategias de supervivencia; Campeche; México.

\section{[en] The expansion of soybean cultivation in Campeche, México: Problematic and perspectives}

\begin{abstract}
The soybean is the grain in which greater food dependency has Mexico, reason why as of 2008, the government has promoted his culture, granting excellent subsidies, as much to producers as to buyers of the grain, thus contributing to a recent process of expansion in certain states, as it happens in Campeche. The objetive of this article is the analysis of the characteristics and effects of those supports, as well as of the rest of factors that until today they have taken to the producers of the mentioned state to initiate or to expand the cultivation of the soybean. The findings of the investigation reveal that although the producers have improved their levels of income, the process is vulnerable, as it depends on variables like the governmental supports, the international prices of the soybean and exchange rate. Although the study of the negative effects of genetically modified soybeans (GM) in other areas (environment, biodiversity, deforestation, human and animal health) is not the purpose of this investigation, some information will be provided, as on the conflict between soybean producers and beekeepers in the state of Campeche.
\end{abstract}

Key words: Soybeans; subsidies; surviving strategies; Campeche; Mexico.

1 Instituto de Geografía. Universidad Nacional Autónoma de México. E-mail: flavia2451@gmail.com 


\title{
[fr] L'Expansion de la Culture du Soja en Campèche, le Mexique: Problématique et Perspectives
}

\begin{abstract}
Résumé. Le soja est le grain dans lequel une plus grande dépendance alimentaire a le Mexique, ce pourquoi à partir de 2008, le gouvernement a promu sa culture, en accordant des subventions significatives, tant à des producteurs comme à des acheteurs du grain, contribuant ainsi à un récent processus d'expansion dans certains états, comme il se produit en Bois de Campèche. Cet article a pour but l'analyse les caractéristiques et les effets de ces appuis, ainsi que du reste de facteurs que jusqu'à aujourd'hui aux producteurs de l'état mentionné à entamer ou ils ont développé la culture du soja. Les découvertes de la recherche révèlent que bien que les producteurs ils ont amélioré leurs niveaux de recette, le processus est vulnérable, en dépendant de variables comme les appuis gouvernementaux, les prix internationaux du soja et le taux de change. Bien que l'étude des effets négatifs de soja génétiquement modifiées (GM) dans d'autres domaines (environnement, la biodiversité, la déforestation, la santé humaine et animale) ne sont pas le but de cette recherche, certaines informations seront fournies, comme sur le conflit entre les producteurs et les apiculteurs de soja que l'état de Campeche.
\end{abstract}

Mots clés: Soja; subventions; stratégies de survie; Campeche; Le Mexique.

Cómo citar. Echanove Huacuja, F. (2016): La expansión del cultivo de la soja en Campeche, México: Problemática y perspectivas. Anales de Geografía de la Universidad Complutense, 36(1), 49-69.

Sumario. 1. Introducción. 2. La dependencia de México de la soja importada. 3. Inicio y difusión del cultivo de la soja en Campeche. 4. La soja, una alternativa productiva para los menonitas. 5. El ejido Carlos Cano Cruz. 6. Causas de la expansión de la soja en Campeche. 7. Reflexiones finales. 8. Bibliografía. 9. Entrevistas realizadas.

\section{Introducción}

El frijol o poroto de soja (al cual llamaremos posteriormente solo soja) es una leguminosa (Glycine max) nativa del este de Asia, que ha sido cultivada en China por más de 5.000 años. Ha presentado una relevante expansión a nivel mundial durante las últimas décadas; tan solo entre 1991 y 2014 la producción de soja casi se triplicó, incrementándose de 108 a 308 millones de toneladas (FAO, 2016). La superficie cultivada con ese grano en el ciclo 2013/2014 fue de 113 millones de hectáreas (USDA, 2016), equivalente al área combinada de Francia, Alemania, Bélgica y los Países Bajos (WWF, 2014). La soja constituye uno de los granos más versátiles, siendo utilizado en la producción de alimentos animales por ser una valiosa fuente de proteína, así como en el consumo humano, tanto como en forma de grano entero, como en diversos productos elaborados (aceite comestible, tofu, leche de soja, margarinas, sustitutos de carne, tempeh y salsa de soja) y, más recientemente, en barras nutricionales, cereales, pasta, panes y quesos, entre otros. La soja también es usada en la fabricación de productos industriales como lubricantes, plásticos, ceras y diversos químicos como los ácidos grasos, así como en la elaboración de biocombustible. Un derivado de la soja, la lecitina, es utilizado como emulsionante en una amplia gama de alimentos procesados como chocolates, helados y alimentos horneados. Sin embargo, la mayoría de la producción mundial de ese grano (75\%) se utiliza en la elaboración de forraje animal (WWF, 2014; Soy Australia LTD, 2011). 
Estados Unidos, Brasil y Argentina producen en promedio el $80 \%$ de la soja mundial. En el caso de México, su reducida producción en relación al consumo doméstico ha hecho que las compras externas sean muy relevantes, ubicando a ese país en el cuarto sitio como importador, antecedido por la Unión Europea, China y Japón (USDA, 2015a). La soja es el grano en el que mayor dependencia alimentaria tiene México, ya que importa el 95\% de su consumo. La creciente demanda de este grano ha obedecido sobre todo a la expansión en el consumo de productos pecuarios (carnes, lácteos y huevos), el cual durante ciertas décadas ha sido mucho mayor en los países en desarrollo que en los desarrollados, como efecto del crecimiento de la población, la urbanización y el incremento en los ingresos (Delgado, 2003).

A partir de 2007, el gobierno mexicano ha tratado de impulsar la producción de soja, otorgando subsidios a productores y compradores del grano a través de dos programas de apoyo (Pro-Oleaginosas y Agricultura por Contrato). Ello ha contribuido notablemente a la expansión del cultivo en ciertas regiones, como en la Península de Yucatán, particularmente en el estado de Campeche. El objetivo de nuestra investigación es analizar los distintos elementos o causas que han motivado a los productores de dicho estado a iniciar o expandir el cultivo de soja, lo que, por supuesto implica el estudio de las características y efectos de los mencionados programas de apoyo. Buena parte de la soja cultivada en Campeche es genéticamente manipulada (GM) y, aunque nuestro análisis no se centra en sus efectos en la salud humana, el medio ambiente y los apicultores, se dará información proporcionada por los productores (entrevistas directas) en cuanto a su preferencia por cultivar ese tipo de semilla sobre la llamada "convencional”.

Un hallazgo de la presente investigación es el hecho de que el cultivo de soja ha representado para los productores una estrategia de supervivencia, por lo que nuestro análisis será guiado por el enfoque de supervivencia, crecientemente adoptado por los geógrafos del desarrollo, y que contribuye a un mejor entendimiento del desarrollo local mediante el análisis de las respuestas de la gente ante los desafíos globales (De Hann y Zoomers, 2003: 350). La relación dialéctica entre el concepto de supervivencia y el de lugar ha sido enfatizada por Bebbington (2003: 302), quien indica que los lugares son producto de las estrategias de supervivencia de la gente, al mismo tiempo que elementos estructurales de dicha supervivencia. Para Long (2001: 54), supervivencia expresa la idea de individuos y grupos luchando por subsistir, enfrentándose a incertidumbres, respondiendo a nuevas oportunidades y haciendo elecciones entre diferentes posiciones de valor. Dicho autor señala (2001: 234) que los cambios en los hábitos de consumo, el desarrollo tecnológico, las estrategias de las empresas transnacionales y de las grandes cadenas de autoservicio, dan lugar a toda una gama de efectos o repercusiones que afectan significantemente el tipo de decisiones tomadas por las unidades familiares agrícolas. Sin embargo, para Wallman (1982: 5), las estrategias de supervivencia no tienen que ver solamente con aspectos materiales o de recursos económicos, sino que incluyen percepciones, habilidades, simbolismos y estrategias organizacionales. En este mismo sentido, Long (2001: 53), enfatiza que los mecanismos de supervivencia van más allá de las estrategias 
económicas a nivel de las unidades familiares o inter-familiares, teniendo que ver con elecciones de valor, status y sentido de identidad.

\section{La dependencia de México de la soja importada}

En el período 1990-94, México alcanzó su mayor producción histórica de soja (en 1991 fue de casi 725.000 toneladas), no obstante lo cual continuó dependiendo de importaciones para cubrir su consumo doméstico (Tabla 1).

Tabla 1. México: producción e importación de soja

\begin{tabular}{|c|c|c|c|c|c|c|}
\hline Años & $\begin{array}{c}\text { Superficie } \\
\text { cosechada (ha) }\end{array}$ & $\begin{array}{l}\text { Producción } \\
\text { (toneladas) }\end{array}$ & $\begin{array}{l}\text { Rendimientos } \\
\text { (ton/ha) }\end{array}$ & $\begin{array}{l}\text { Importaciones } \\
\text { soja grano } \\
\text { (ton) }\end{array}$ & $\begin{array}{c}\text { Importaciones } \\
\text { pasta y harina } \\
\text { de soja (ton) }\end{array}$ & $\begin{array}{c}\text { Importaciones } \\
\text { aceite de soja } \\
\text { (ton) }\end{array}$ \\
\hline 1990 & 285.615 & 575.366 & 2,01 & 834.075 & 264.684 & 2.657 \\
\hline 1991 & 341.679 & 724.969 & 2,12 & 1.481 .434 & 349.127 & 19.296 \\
\hline 1992 & 322.576 & 593.540 & 1,84 & 1.914 .113 & 496.859 & 28.247 \\
\hline 1993 & 237.765 & 497.566 & 2,09 & 1.758 .386 & 255.695 & 33.130 \\
\hline 1994 & 288.499 & 522.583 & 1,81 & 2.073 .116 & 432.937 & 22.766 \\
\hline 1995 & 134.396 & 189.774 & 1,41 & 2.017 .558 & 365.082 & 61.441 \\
\hline 1996 & 49.064 & 56.074 & 1,14 & 2.647 .875 & 295.826 & 57.320 \\
\hline 1997 & 122.548 & 184.526 & 1,51 & 3.093 .385 & 147.351 & 93.848 \\
\hline 1998 & 94.065 & 150.296 & 1,60 & 3.099 .834 & 147.547 & 104.043 \\
\hline 1999 & 81.159 & 132.824 & 1,64 & 3.287 .211 & 309.493 & 99.405 \\
\hline 2000 & 69.969 & 102.314 & 1,46 & 3.436 .483 & 155.529 & 105.688 \\
\hline 2001 & 73.725 & 121.671 & 1,65 & 3.941 .765 & 284.452 & 95.596 \\
\hline 2002 & 56.473 & 86.499 & 1,53 & 3.919 .975 & 445.556 & 216.865 \\
\hline 2003 & 67.880 & 126.006 & 1,86 & 3.887 .804 & 284.452 & 139.892 \\
\hline 2004 & 88.840 & 133.346 & 1,50 & 2.870 .856 & 833.140 & 105.615 \\
\hline 2005 & 96.061 & 187.016 & 1,95 & 3.440 .515 & 1.360 .480 & 156.139 \\
\hline 2006 & 54.211 & 81.113 & 1,50 & 3.742 .780 & 1.709 .161 & 108.066 \\
\hline 2007 & 62.580 & 88.371 & 1,41 & 3.663 .134 & 1.639 .870 & 173.292 \\
\hline 2008 & 75.767 & 153.022 & 2,02 & 3.550 .054 & 1.467 .741 & 246.923 \\
\hline 2009 & 64.740 & 120.942 & 1,87 & 3.281 .264 & 1.353 .320 & 177.491 \\
\hline 2010 & 153.473 & 167.666 & 1,09 & 3.586 .833 & 1.395 .545 & 212.191 \\
\hline 2011 & 155.512 & 205.234 & 1,32 & 3.158 .106 & 1.451 .334 & 154.843 \\
\hline 2012 & 142.329 & 247.500 & 1,74 & 3.297 .985 & 1.375 .574 & 168.533 \\
\hline 2013 & 157.419 & 239.248 & 1,52 & 2.733 .295 & 1.291 .920 & 182.497 \\
\hline
\end{tabular}

Nota: las importaciones son la procedentes de Estados Unidos.

Fuente: SAGARPA (2015a); USDA (2015b) y SEGOB (2013).

Posteriormente, y después de años de una tendencia decreciente tanto en superficie cosechada como en producción, a partir de 2009 estas variables empiezan a repuntar, aunque sin alcanzar los primeros niveles señalados. En 2013, por ejemplo, se produjeron 239.248 toneladas, las cuales solo cubrieron el 5\% del consumo doméstico (grano, harinas y aceite de soja), el cual fue de 4,4 millones de toneladas, siendo esta oleaginosa, como ya comentamos, el grano en el que mayor dependencia externa tiene México. Esta se profundizó durante los catorce años del período de vigencia del TLCAN (Tratado de Libre Comercio de América del Norte), durante el cual las importaciones de soja (procedentes casi totalmente de Estados Unidos) se duplicaron, 
al pasar entre 1994 y 2008 de 2,5 a 5,2 millones de toneladas (Tabla 1). Estas compras fueron incentivadas por los precios subsidiados (“dumping”) a los que ingresó la soja al mercado mexicano; para el período 1994-2005, Wise (2011: 48) ubica en $12 \%$ el porcentaje de dicho dumping, entendido éste como la diferencia entre el costo de producción y el precio de venta del grano. A esto hay que añadir que México eliminó completamente los aranceles a la importación de soja en 2003, en el marco del Tratado señalado (Yúnez, 2010: 29).

La dimensión de las compras externas ha convertido a México en el segundo país comprador de soja grano de Estados Unidos y en el primero de pasta de soja y aceites (USDA, 2015c). Para los productores mexicanos, las posibilidades de competir con la soja estadounidense eran muy reducidas; tan solo entre 1994 que inicia el TLCAN y 1995, las superficies cosechadas de soja en México se desplomaron a menos de la mitad (Tabla 1). Pero también otras medidas de política agrícola desincentivaron la producción doméstica de soja y ocasionaron la reubicación geográfica de su cultivo, como fue la desaparición en 1989 de su precio de garantía (precio mínimo de venta fijado por el gobierno), al igual que ocurrió con los otros granos, a excepción del maíz y frijol, cuyos precios de garantía continuaron hasta 1995. Esto, aunado a los subsidios gubernamentales otorgados al cultivo del maíz, contribuyó a que muchos productores sustituyeran la soja por el maíz, como ocurrió en los estados de Sinaloa y Sonora, que aportaban hasta 1994 el 90\% de la producción doméstica, año a partir del cual inicia el desplome de este cultivo (SAGARPA, 2015a).

La mencionada expansión del cultivo de soja a partir de 2009 se ubica en los que hoy son los principales estados productores, cuya importancia en 2013 fue la siguiente: Tamaulipas (117.729 toneladas), San Luis Potosí (38.097 toneladas), Campeche (33.025 toneladas) y Chiapas (29.047 toneladas) (SAGARPA, 2015a). Dicho grano se cultiva en el ciclo primavera-verano, y en su mayoría es de secano; en 2013, por ejemplo, solo se irrigó el 17\% de la superficie cosechada en México, la cual se ubicó mayoritariamente en el estado norteño de Tamaulipas. En el caso de Campeche, que analizaremos en el siguiente apartado, solo se censaron en ese año 370 hectáreas de riego (2\% del total cultivado).

\section{Inicio y difusión del cultivo de la soja en Campeche}

Las primeras siembras de soja en Campeche, de acuerdo a cifras oficiales, datan de 1980. Durante los últimos diez años (2003-2013), la superficie cosechada de ese grano y su producción anual han sido muy cambiantes, sin embargo, la tendencia general de ambas variables ha sido creciente. En ese período, la superficie cosechada pasó de 650 a 15.634 hectáreas, lo que, aunado al incremento de casi el doble de los rendimientos, motivó que la producción aumentara de 715 a 33.025 toneladas (Tabla 2 y Figura 1). Para 2014, la superficie sembrada casi se duplicó (29.200 hectáreas) (Chi-Bonilla, 2015, entrevista directa). Esta expansión de la soja, un hecho 
relativamente nuevo, se ha dado mediante el desmonte de terrenos con vegetación autóctona, pero también a través de la sustitución del cultivo del maíz por el de esa oleaginosa, por las razones que daremos posteriormente.

Tabla 2. Campeche: evolución del cultivo de soja

\begin{tabular}{|c|c|c|c|}
\hline Años & $\begin{array}{c}\text { Superficie } \\
\text { cosechada (ha) }\end{array}$ & $\begin{array}{c}\text { Rendimientos } \\
\text { (ton/ha) }\end{array}$ & $\begin{array}{c}\text { Producción } \\
\text { (ton) }\end{array}$ \\
\hline 2003 & 650 & 1,10 & 715 \\
\hline 2004 & 1.772 & 1,85 & 3.284 \\
\hline 2005 & 5.545 & 2,58 & 14.314 \\
\hline 2006 & 3.876 & 1,52 & 3.876 \\
\hline 2007 & 1.862 & 2,06 & 3.843 \\
\hline 2008 & 5.252 & 2,18 & 11.447 \\
\hline 2009 & 11.208 & 2,18 & 24.457 \\
\hline 2010 & 17.660 & 1,43 & 25.229 \\
\hline 2011 & 10.727 & 2,69 & 28.879 \\
\hline 2012 & 6.794 & 2,48 & 16.880 \\
\hline 2013 & 15.634 & 2,11 & 33.025 \\
\hline
\end{tabular}

Fuente: SAGARPA (2015a).

Figura 1. Campeche: superficie cosechada y producción de soja

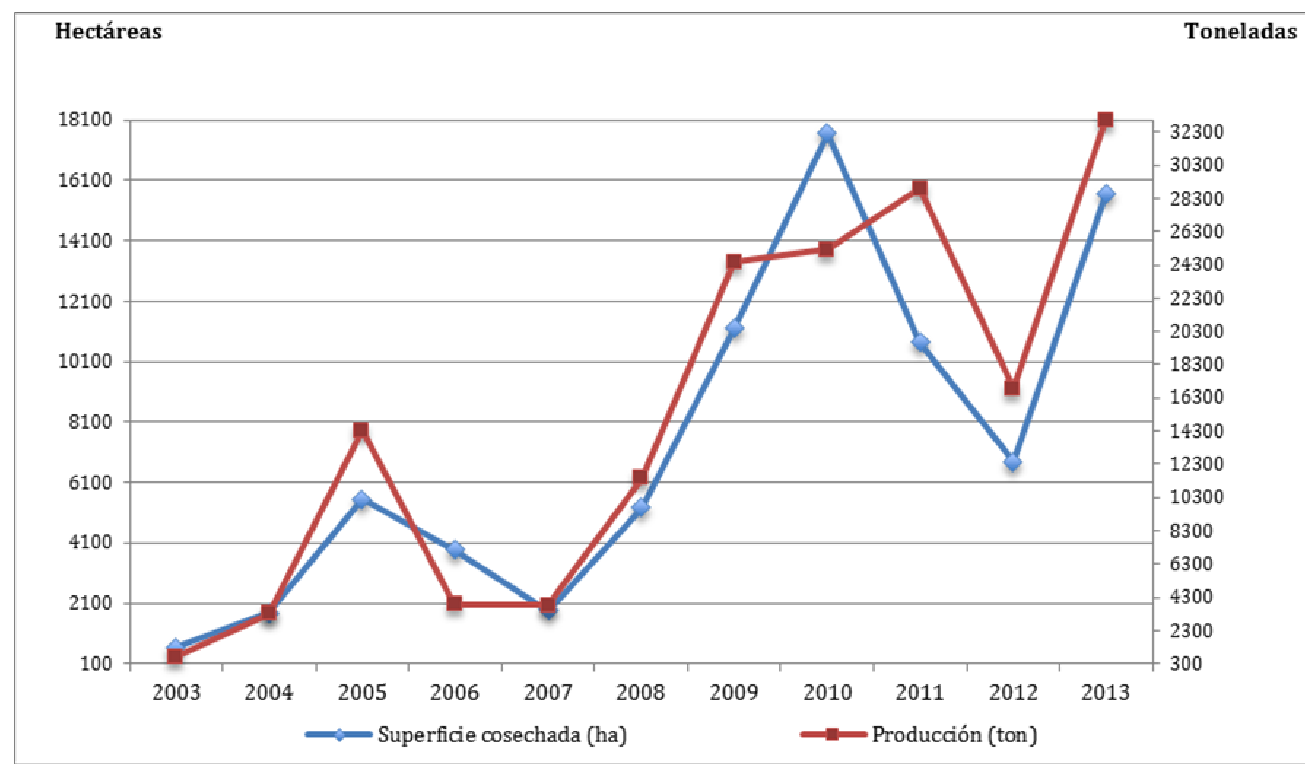

Fuente: SAGARPA (2015a). 
$\mathrm{Al}$ ser la soja de Campeche un cultivo mayoritariamente de secano, es vulnerable a eventos climáticos como las sequías. Pero también es afectada por huracanes o ciclones, y por el exceso de lluvias, como ocurrió en 2013, en que se presentaron durante la fase de la cosecha, dificultando esta labor que es totalmente mecanizada, además de verse afectada la calidad del grano obtenido. La soja se siembra en juniojulio, y se cosecha en noviembre-diciembre, siendo los principales municipios productores Campeche y Hopelchén, que aportan casi el 90\% de la producción del estado (Tabla 3 y Mapa 1).

Tabla 3. Campeche: producción de soja por municipios (2013)

\begin{tabular}{cccccc}
\hline Municipio & $\begin{array}{c}\text { Superficie } \\
\text { Cosechada } \\
(\mathrm{Ha})\end{array}$ & $\begin{array}{c}\text { Producción } \\
(\text { Ton })\end{array}$ & $\begin{array}{c}\text { Rendimiento } \\
(\text { Ton/Ha })\end{array}$ & $\begin{array}{c}\text { PMR* } \\
(\$ / \text { Ton })\end{array}$ & $\begin{array}{c}\text { Valor } \\
\text { Producción } \\
\text { (Miles de Pesos) }\end{array}$ \\
\hline Campeche & 7.647 & 16.239 & 2,52 & 6.500 & 125.057 \\
Hopelchén & 6.851 & 12.123 & 1,77 & 6.500 & 78.799 \\
Champotón & 1.016 & 1.464 & 1,44 & 6.492 & 9.509 \\
Tenabo & 100 & 122 & 1,22 & 6.200 & 756 \\
Escárcega & 20 & 76 & 3,80 & 4.300 & 327 \\
Total & $\mathbf{1 5 . 6 3 4}$ & $\mathbf{3 3 . 0 2 5}$ & $\mathbf{2 , 1 1}$ & $\mathbf{6 . 4 9 3}$ & $\mathbf{2 1 4 . 4 4 8}$ \\
\hline
\end{tabular}

* Precio medio rural.

Fuente: SAGARPA (2015a).

Aunque como ya señalamos, el propósito de esta investigación no es el ahondar sobre los tipos de semillas de soja utilizados y sus diversos efectos, sí daremos algunos datos generales, necesarios para comprender la expansión del cultivo de ese grano, así como las limitantes o problemática que se ha presentado en los últimos años. En Campeche se han sembrado dos tipos de semillas, la llamada Huasteca o "convencional" (no genéticamente manipulada), producida en el estado de Tamaulipas por el organismo gubernamental INIFAP (Instituto Nacional de Investigaciones Forestales, Agrícolas y Pecuarias), y la genéticamente manipulada o transgénica (GM), producida y distribuida mayoritariamente por Monsanto. Este grano pertenece al grupo de los llamados Roundup Ready, que son resistentes a la aplicación del herbicida denominado glifosato, producido también por Monsanto (GRAIN, 2014). La semilla transgénica se empezó a utilizar en el Estado en 2002 (350 hectáreas) y, para 2005, las 4.500 hectáreas que se sembraron con ella representaron ya el $80 \%$ de la superficie total cultivada con soja en Campeche. Desgraciadamente, no existen cifras oficiales de cuánta superficie se ha cultivado con cada tipo de semilla, sin embargo, tanto entrevistas realizadas a firmas distribuidoras de la semilla transgénica (De Matteis, 2013), como el trabajo de campo realizado en 2013-2014 en los principales municipios productores de Campeche reveló que en los últimos años ha prevalecido el uso de la transgénica. Diversos productores entrevistados señalaron preferir este tipo de semilla debido a que se genera mucho menos maleza que con la Huasteca, lo que disminuye el costo de producción, al reducirse el pago de mano de obra, ya que solo es posible eliminar dicha maleza 
manualmente (Olvera, 2013; Valencia, 2013; Tlilayatzi, 2013; Aguilar, 2013; Pérez, 2013). 


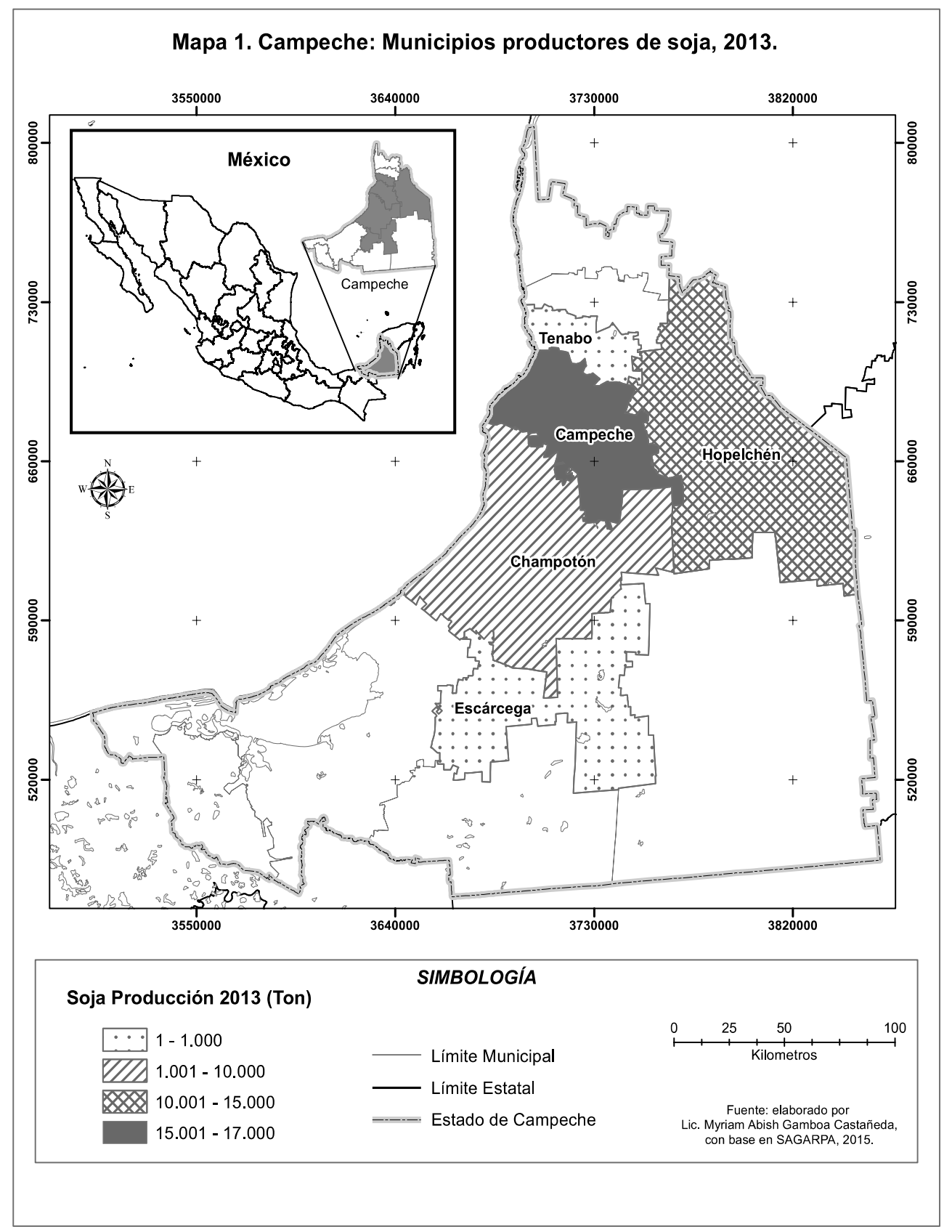

Fuente: Myriam Caboa Castañeda con base en SAGARPA, 2015 
Lo que es innegable es el hecho de que la activa difusión y el constante suministro de semilla por parte de Monsanto ha sido un factor clave en la expansión de la soja de Campeche. Tan es así, que en 2012, en que el gobierno prohibió la siembra de semilla GM en ese Estado, como producto de un amparo legal otorgado a los productores de miel, la superficie sembrada de soja se desplomó (4,000 hectáreas menos que el año anterior), al no haber existido la alternativa de contar con suficiente semilla Huasteca, aunque también diversos productores, acostumbrados ya a la semilla transgénica, prefirieron no sembrar soja en ese año.

\section{La soja, una alternativa productiva para los menonitas}

El 90\% del volumen de soja producido en Campeche proviene de menonitas y, el restante porcentaje, de los que se denominan locales o "mexicanos" (SAGARPA, 2015b; Hernández, 2014). En ese Estado se concentra la mayoría de los menonitas que han llegado a la península de Yucatán; se calcula que hay alrededor de 2.000 familias, lo que equivale a poco más de 14.000 personas. Se organizan en "colonias", conformadas por tierras compradas, por lo que constituyen propiedades privadas. Los primeros menonitas que llegaron a Campeche fundaron en 1987 la colonia Nuevo Progreso y, a partir de entonces, fueron arribando progresivamente menonitas procedentes de otros estados de México (Tamaulipas, Chihuahua, Zacatecas, San Luis Potosí, etc.). Los que vivían en la zona norte del país emigraron buscando mejores condiciones para realizar sus actividades productivas, dado que el entorno de extrema inseguridad que desde hace tiempo se vive en esa zona. Muchos de los residían en el Estado norteño de Tamaulipas, integrantes ahora de colonias como Nuevo Progreso y Las Flores (municipio de Hopelchén), eran productores de soja, por lo que traían el conocimiento del cultivo de ese grano (Wall, 2014, entrevista directa).

En el municipio de Campeche, el principal productor del Estado, la mayor parte de la soja se cultiva en tierras de los ejidos Carlos Cano Cruz y San Luciano y en la propiedad privada Los Laureles (no menonita). Sin embargo, la gran mayoría de ese grano es producido por menonitas que residen en el vecino municipio de Hopelchén, sobre todo mediante la renta de tierras a dichos ejidatarios y productores privados. En 2013, por ejemplo, 9 ejidatarios de Carlos Cano cultivaron soja, 7 del ejido San Luciano y 11 propietarios privados de Los Laureles, es decir, solamente 27 productores no menonitas (Canul, 2014, entrevista directa). En el segundo municipio sojero, Hopelchén, es donde se ubica la mayor parte de las colonias menonitas que producen ese grano, cuyos integrantes cultivaron en 2013 alrededor del 80\% de las 6.851 hectáreas de soja cosechadas en el municipio. Entre dichas colonias, destacan las de Las Flores y La Nueva Trinidad, que producen la mayoría de la soja de Campeche. En segundo término en importancia figura la propiedad privada El Yibel (municipio de Hopelchén), de Jacobo Xacur, empresario yucateco de origen libanés, y en la cual se cultiva anualmente 1.000-1.200 hectáreas de soja (primavera-verano), y en las cuales se siembra posteriormente maíz (ciclo otoño-invierno). Los 
rendimientos de ambos cultivos son de los mejores del Estado, en la soja promedian 3 ton/ha, y en maíz obtienen hasta 8-9 ton/ha (Canul, 2014).

En resumen, en los dos principales municipios sojeros, solamente 30 productores no son menonitas, lo que representa solo el 15\% del total de los 200 que cultivan ese grano en Campeche. Junto a los existentes en los otros municipios productores, contribuyen, como ya señalamos, tan solo al $10 \%$ de la producción estatal de soja. Cabe mencionar que también son los menonitas los que producen alrededor del $70 \%$ de la producción de maíz de Campeche (Escalante, 2013, entrevista directa).

Es interesante señalar las particularidades del régimen de propiedad y de la distribución de las superficies sembradas de soja entre los menonitas que, por cierto, son las mismas que con el resto de los cultivos comerciales que emprenden. La mayoría de las tierras que cultivan se encuentran fuera de sus respectivas colonias, constituyendo parcelas o terrenos pertenecientes a ejidos $\mathrm{y}$, en menor grado, a propiedades privadas, ubicados tanto en el mismo municipio donde se asientan las colonias, como en otros vecinos o más distantes. El mecanismo más utilizado por parte de los menonitas es, como ya mencionamos, el arrendamiento de tierras, aunque en el caso de la soja existen también otros acuerdos que reseñaremos más adelante.

En la colonia Las Flores, fundada en 1999 por 65 familias menonitas, el maíz había sido el cultivo mayoritario, pero en 2003 empezaron a sembrar soja, apoyados en la experiencia que tenían como previos productores de este grano en Tamaulipas. En 2013 sembraron en Campeche 5.000 hectáreas de soja (Classen, 2014; Huiber, 2014, entrevistas directas), por lo que esta colonia es la principal productora de ese grano en la entidad. Sin embargo, de esa superficie, solamente 500 hectáreas se ubican dentro de la colonia, el resto son parcelas arrendadas fuera de ella (Harder, 2014, entrevista directa), tanto a los ejidos cercanos a su colonia, como a los ubicados en otros municipios (Campeche y Champotón). Algunos menonitas han podido incluso comprar tierras, sin embargo, debido a la gran demanda de ellas por parte del conjunto de colonias de Campeche, incluso la tierra para arrendar (ya desmontada) es escasa, por lo que algunos están alquilando terrenos con "acahuales" (vegetación forestal común en el estado), los cuales desmontan para su cultivo gracias a que tienen la maquinaria necesaria (Harder, 2014, entrevista directa). Esta práctica, realizada por la generalidad de las colonias menonitas, ha suscitado diversas críticas por parte no solo de grupos ambientalistas, que denuncian el grave proceso de deforestación que dichos productores están llevando a cabo no solo en Campeche, sino también en el vecino Estado de Yucatán (Tribuna, 2014a; 2014b).

La segunda colonia productora de soja, La Nueva Trinidad, fundada en 1996, también se conformó mediante la compra de tierras. En un inicio se dedicaba mayoritariamente al cultivo del maíz, pero durante los últimos diez años sus colonos han ido produciendo cada vez más soja; en 2012 sembraron 3.500 hectáreas de ese grano, cifra que subió a 4.500 hectáreas en 2013 (Canul, 2014, entrevista directa). La colonia que le sigue en importancia, Nuevo Progreso, se integra por 350 productores; solo la mitad de la superficie total que cultivan es de su propiedad (ubicada en los terrenos de la colonia), mientras que la otra se conforma por terrenos rentados fuera 
de ella. Esta productiva colonia es hasta hoy mayoritariamente maicera, grano del cual cosecharon 7.000 hectáreas en 2013. Es de las más nuevas como productora de soja, ya que empezaron a sembrarla en 2011; para 2013 cosecharon 500 hectáreas (34 productores) de ese grano, y para 2014 planeaban sustituir maíz por soja en al menos 2.500 hectáreas (Wall, 2014; Canul, 2014, entrevistas directas).

En las tres principales colonias sojeras reseñadas, existe un cierto grado de diversificación productiva, ya que, aparte de maíz y soja, cultivan sorgo, el cual siembran en las mismas parcelas recién cosechadas de soja . Pero también producen sorgo de manera continua en otras superficies, usando el método de no rastrearlo después de la cosecha, logrando así que el grano retoñe, aunque los rendimientos sean menores. La alternativa maíz-sorgo en las mismas superficies es también muy común, logrando así cubrir dos ciclos productivos al año. Algunas colonias cultivan sandía con fines comerciales (La Nueva Trinidad), jitomate de vara, calabaza, etc., además de producir leche (Nuevo Progreso y La Nueva Trinidad), quesos (colonia Yalnón), y hortalizas con fines de autoconsumo.

La mayoría de los productores menonitas son medianos (dentro de los parámetros de México), es decir, cultivan entre 80 y 200 hectáreas, pero los hay grandes, como en La Nueva Trinidad, donde algunos productores detentan 200-400 hectáreas sólo de soja, y hasta 800 hectáreas, como en Las Flores. En dos colonias, Nuevo Progreso y La Nueva Trinidad, existen organizaciones formales, ya que cada una cuenta con dos SPR (Sociedades de Producción Rural), lo que les facilita, entre otras cosas, la adquisición de insumos y crédito. Por ejemplo, la SPR Agroservicios La Nueva Trinidad, en la colonia de ese nombre, agrupa a 60-70 productores, lo que les ha permitido acceder a financiamiento gubernamental para la construcción de los 3 silos (depósitos de granos) con los que cuentan. Sin embargo, en la principal colonia sojera, Las Flores, se produce y comercializa individualmente, y no cuentan con infraestructura para almacenar granos.

Hemos señalado ya que la producción de soja está sustentada en gran parte en el arriendo de tierras a ejidos. Aunque no se cuentan con cifras sobre el monto de la superficie arrendada, los datos obtenidos en las tres principales colonias menonitas nos da una idea. En algunos ejidos, las superficies en arriendo representan el 80-90\% del total de las tierras de cultivo, como es el caso del ejido Chencoh (municipio de Holpechén), donde son los colonos de Las Flores los que las cultivan. A estos mismos colonos les arriendan la mayor parte de sus tierras los ejidos ubicados en la región de las sabanas (municipio de Champotón) (Harder, 2014; Canul, 2014, entrevistas directas). Aunque en menor proporción, como ya indicamos, diversos ejidos del municipio de Campeche (Pich, Carlos Cano Cruz, San Luciano, etc.), y la propiedad privada Los Laureles, rentan parte de sus tierras a los menonitas. La pregunta es ¿por qué los ejidatarios prefieren rentar la tierra a cultivar ellos mismos la soja? De acuerdo a las diversas entrevistas realizadas, tanto en los dos últimos ejidos mencionados, como en Los Laureles, la limitante fundamental que tienen sus integrantes es la falta de maquinaria. A diferencia de otros cultivos como el maíz, la soja requiere forzosamente de maquinaria en diversas etapas del cultivo, sobre todo en la siembra y cosecha. Cuando el grano está listo para cosecharse, tiene que hacerse 
esta labor con brevedad, porque de no ser así, se corren riesgos, como el que la vaina se abra y se caiga el grano al suelo. Las cosechadoras utilizadas tienen que contar con adaptaciones especiales para cosechar la soja. Sin embargo, y a diferencia de los menonitas, son pocos los ejidatarios que cuentan con dicha maquinaria. Los pocos que aun careciendo de ella deciden cultivar soja, tienen que pagar a productores menonitas para que les hagan las labores mecanizadas, cuestión que significa un riesgo, sobre todo en la cosecha, dado que obviamente dichos productores primero atienden sus propios cultivos y luego acuden a los de los ejidatarios. Los menonitas que no tienen maquinaria propia no cultivan soja, se dedican al maíz y sorgo.

Aledañamente, el ingreso obtenido por el arriendo de tierra es relevante para los ejidatarios. En 2014, por ejemplo, el precio de la renta por hectárea estaba en \$1.500 $2.000(\mathrm{MXN})$ por un ciclo productivo (6 meses), pero la mayoría de los ejidatarios rentan su tierra durante todo el año. Los menonitas tratan de hacer contratos de renta con los ejidatarios por el mayor tiempo posible (entre 8 y 15 años o, al menos, 2 años). Otro acuerdo que es menos común, pero que hasta hoy día se hace, se denomina "al porcentaje", y consiste en que el menonita le entrega al ejidatario por el uso de sus tierras entre un 10 y $20 \%$ del ingreso obtenido por la venta del grano cosechado. Sin embargo, diversos ejidatarios señalaron que este acuerdo no les conviene, ya que si el cultivo es afectado por alguna eventualidad, ellos arriesgan sus ingresos (Valencia, 2013, entrevista directa). A continuación, reseñaremos el caso de uno de los principales ejidos rentistas, para ahondar más en las causas de esta práctica.

\section{El ejido Carlos Cano Cruz}

El ejido Carlos Cano Cruz (municipio de Campeche) es conocido como "Los Tlaxcaltecas" porque la mayoría de sus integrantes provenían de ese lejano Estado, donde, o no tenían tierra para cultivar, o ésta era insuficiente, ante lo cual la gobernadora de Tlaxcala (Beatriz Paredes) y después líder de la CNC (Confederación Nacional Campesina), hizo un convenio con el gobernador de Campeche para ubicar a los solicitantes o colonos interesados, aprovechando el programa de colonización de este Estado (Valencia, 2013; Suárez, 2005: 44). En 1990, la asamblea de colonos otorgó 5 hectáreas de tierra para ser cultivada por cada uno de los 92 solicitantes. Con diversos apoyos del gobierno de Tlaxcala (alimentos, transporte, maquinaria pesada, etc.), los colonos fueron sobreviviendo y desmontaron los terrenos que cada uno eligió. Durante ese año y el siguiente, el grupo de migrantes trabajó colectivamente la superficie laborable, ya que ésta no estaba parcelada ni tenían títulos individuales de propiedad, lo cual generó conflictos entre ellos (Valencia, 2013, entrevista directa). A mediados de 1991, el gobierno de Tlaxcala, a decir de los colonos, "los dejó a su suerte”, ya que suspendió los apoyos que otorgaba, con lo cual sus condiciones de vida se deterioraron (Suárez, 2005: 52). Esto, aunado a la inexperiencia como agricultores de muchos de ellos, su falta de maquinaria y las extremas temperaturas, hicieron que varios colonos regresaran a su estado de origen (Valencia, 2013). 
Entre 1992 y 1993 la asamblea de colonos decidió incrementar en 18 hectáreas más la superficie individual laborable (a un total de 23 hectáreas), la cual fue elegida por cada colono, deslindada y, por primera vez, cultivada de manera individual. En enero de 1993 se constituyó oficialmente el Centro de Población Ejidal Carlos Cano Cruz, dotando a 92 solicitantes con 9.652 hectáreas, la mayoría de ellas de monte con selva media o acahuales, y un 40\% susceptible a cultivos de temporal (Suárez, 2005). Sin embargo, y como ya mencionamos, varios de los 92 solicitantes ya se habían ido y nunca reclamaron sus derechos ejidales. En 1996, el ejido se incorpora al PROCEDE (Programa de Certificación de Derechos Ejidales y Titulación de Solares Urbanos) y, en 1998, los 56 ejidatarios que quedaban obtuvieron sus certificados parcelarios, derechos sobre las tierras de uso común y títulos de propiedad de sus solares (Valencia, 2013). A cada uno de éstos se le dotó de 150 hectáreas de terreno (incluyendo las 23 hectáreas que ya tenían deslindadas), de las cuales 92 hectáreas eran laborables, superficie muy superior a la media nacional. Cada ejidatario cuenta hoy con un promedio de 2,9 parcelas (Suárez, 2005: 69).

Es a partir del momento en que se regularizan las tierras cuando empiezan a llegar los menonitas, en su mayoría de la cercana colonia Las Flores, para establecer acuerdos con los ejidatarios. A éstos les ofrecieron desmontar sus tierras, dado que sólo aquéllos contaban con la maquinaria necesaria, a cambio de cultivarlas libremente durante 5-6 años, es decir, sin ningún pago de por medio. Al término de este tiempo, dichas tierras serían restituidas a sus dueños, acuerdo que siempre fue cumplido (Valencia, 2013). Pero ya con sus tierras desmontadas, muchos ejidatarios empezaron a arrendárselas a los menonitas, interesados en seguirlas cultivando, o bien establecieron con ellos relaciones de tipo mediería, como el ya reseñado acuerdo al porcentaje. Al principio, los menonitas cultivaron solo maíz, pero a partir de 2007 empezaron con la soja.

En 2013, de las 3.000 hectáreas cosechadas de soja en el ejido Cano Cruz, 2.000 fueron cultivadas por los menonitas, mientras que las restantes 1.000 por 9 ejidatarios (Valencia, 2013). Estos alternan dicho grano con el sorgo, hecho común en toda la región. En general, los ejidatarios que cultivan sus parcelas, que son en promedio de 80-100 hectáreas, están muy diversificados productivamente, ya que siembran maíz, sorgo, calabaza, tomate, sandía, jamaica, etc., además de contar muchos de ellos con animales de traspatio o dedicarse también a la apicultura. Aquellos que deciden arrendar sus tierras obtienen un ingreso que consideran atractivo, pero otros factores que influyen en esta decisión tienen que ver con su edad y con las preferencias de sus hijos. Los ejidatarios que recibieron originalmente la tierra tienen más de 50 años de edad, por lo que les es difícil emprender las labores agrícolas si no cuentan con descendientes que los apoyen, pero es muy común, y no sólo en este ejido, que los hijos prefieran ir a trabajar como jornaleros en los campos menonitas, ganando 200 pesos mexicanos (MXN) por jornada-día, que trabajar la tierra del padre, en donde su labor no es siempre retribuida monetariamente (Valencia, 2013). Otros ingresos de la familia ejidal proceden de los apoyos gubernamentales de los programas PROCAMPO y Oportunidades, así como del obtenido en actividades extraparcelarias. Entre éstas, Suárez (2005: 61) encontró el pequeño comercio, albañilería, 
panadería, elaboración de artesanías, plomería, tractorista, técnico de radio y promotor rural.

\section{Causas de la expansión de la soja en Campeche}

Lo reseñado hasta aquí permite entender los diversos factores que han influido en la expansión de la soja. Para empezar, está la presencia de los menonitas, con la maquinaria necesaria y la disposición y experiencia para emprender el cultivo. También están las ventajas que la soja tiene sobre el maíz, que han hecho que este grano sea sustituido por el primero. El maíz requiere $1.200-1.300 \mathrm{ml}$ de agua por hectárea durante su ciclo de cultivo, mientras que la soja (Huasteca o GM) necesita solo $500-700 \mathrm{ml}$, por lo que resiste mejor la sequía, lo que es fundamental en el entorno de agricultura de secano que prevalece en Campeche (De Matteis, 2013, entrevista directa). Diversos productores señalaron también que la soja es menos afectada por el exceso de humedad que el maíz. Otro elemento que ha sido relevante son los precios de venta: los de la soja han sido superiores a los del maíz y mucho más estables. Esta diferencia se ha acentuado durante los últimos cuatro años, en que el precio internacional del maíz ha ido a la baja, arrastrando con ello a los precios en el mercado doméstico. En 2013, por ejemplo, el grueso de los productores de maíz de Campeche recibieron entre 2.300 y 2.400 MXN por tonelada, mientras que la soja se pagó a un promedio de 6,500 MXN por tonelada (Escalante, 2013; Conde, 2014; Canul; 2014; Hernández, 2013; Rempel, 2014). En 2012, los productores de maíz habían recibido un precio promedio mucho mayor (\$3,800 MXN por tonelada), aun así, muy inferior al de la soja, el cual promedió \$7.000 MXN por tonelada (Oliva, 2013, entrevista directa). Y aún aquella minoría de productores de maíz que ingresaron en 2013 al programa de subsidios del gobierno llamado agricultura por contrato, bajo el cual, y a diferencia del mercado libre, reciben por su grano el precio internacional, obtuvieron en promedio 193 USD por tonelada, mientras que la soja se cotizó en 443 USD por tonelada. Un año después, en 2014, los precios de venta del maíz fueron muy similares, y aunque los de la soja decrecieron, siguieron otorgando a los productores mucho mayores ganancias (Chi-Bonilla, 2015).

Claro que los precios tienen que contrastarse con los costos de producción. Aunque éstos son muy variables en función de lo que el productor quiera o pueda invertir, en 2013 los del maíz promediaron 6,000 MXN por hectárea, mientras que en soja esa cifra fue de \$6.500-7.000 MXN por hectárea (De Matteis, 2013, entrevista directa). Es decir, los costos de la soja son un poco superiores a los del maíz, y el rendimiento promedio del primer grano (2,5-3 ton/ha) es menor al del maíz (3-3,5 ton/ha), pero la gran diferencia en sus precios de venta provoca mayores ganancias por cultivar soja (al menos podrían cifrarse en \$2.500 MXN más por cada tonelada) (Valencia, 2013).

Otra ventaja crucial en el caso de la soja es que, a diferencia del maíz, tiene un mercado asegurado. Un reducido porcentaje de los productores maiceros de 
Campeche venden su grano a la principal empresa fabricante de harina de maíz en México, es decir, Maseca, y/o a la firma Sumasa, que abastece a la industria del nixtamal (fabricación de tortillas). Sin embargo, la mayoría de los productores dependen de los intermediarios o acopiadores locales para vender su maíz, y éstos no representan un mercado fiable y constante, además de que los precios ofrecidos a los productores son en general muy bajos, aprovechando la necesidad que éstos tienen de vender, y su carencia de infraestructura de almacenaje.

En contraste, el 90\% de la soja de Campeche y, en general, de la península de Yucatán, es adquirida por la empresa Proteínas y Oleicos, mientras que el 10\% restante lo compra la firma avícola Crío, ambas ubicadas en el Estado de Yucatán (Escalante, 2013). Proteínas y Oleicos, fundada en 1949 por el ya mencionado empresario Jacobo Xacur, muele cada año en su planta de Mérida, Yucatán, alrededor de 325.000 toneladas de granos, conformadas en un $72 \%$ por soja y, el resto, por canola. Alrededor del $90 \%$ de los granos que ingresan a su planta es importado, fundamentalmente de Estados Unidos y, en menor medida, de Paraguay (soja) y Canadá (canola), llegando por mar al puerto de Progreso, Yucatán (Hernández, 2013, entrevista directa). El restante porcentaje lo ha adquirido, desde 2008, en la Península de Yucatán; en 2013, la firma compró en esta región 31.000 toneladas de soja producida por 348 abastecedores (personas físicas y morales), de los cuales 277 se ubicaron en el Estado de Campeche y aportaron el 94\% (29.277 toneladas) de las compras mencionadas. Del estado de Yucatán, 7 productores aportaron 919 toneladas y, de Quintana Roo, 64 productores abastecieron 804 toneladas (Martínez, 2014, entrevista directa). Del total de productores que abastecen a Proteínas y Oleicos, el 90\% son menonitas (Hernández, 2013).

De la soja que ingresa en Proteínas y Oleicos se aprovecha todo, el aceite, las harinas y la cáscara. Esta última es adquirida por la industria que fabrica alimentos animales (aves, cerdos, bovinos, peces, mascotas), la cual es también la compradora mayoritaria de la harina o pasta de soja, principal producto de dicha empresa (74\% del volumen producido). Otro destino de la pasta es el consumo humano, ya que con ella se fabrica soja texturizada para sustitutos de carne o el llamado gluten. El aceite refinado (20\% de su producción) se destina mayoritariamente al mercado doméstico en forma de aceite de cocina, aunque también es comprado como ingrediente de un gran número de productos (galletas, pan, margarinas, chantilly, batidos, helados, chocolates, quesos, leche, tofu, etc.), que utilizan la grasa vegetal en sustitución de la animal, entre otras cosas, por su menor costo. El aceite es también utilizado para frituras (botanas, pollo, pescado, etc.) y otros diversos productos (mayonesas, salsa de soja, chiles enlatados, frijoles y atunes). La fabricación de plásticos, pinturas de aceite, vacunas y cosméticos también usan dicho aceite como ingrediente (Martínez, 2014). Entre los principales compradores de la pasta o harina de soja están las firmas Bachoco (pollo y huevo) y Kekén (puercos) y, de aceite, la empresa Bimbo, principal fabricante de pan industrial en México.

Proteínas y Oleicos ha participado directamente en la promoción del cultivo de soja entre los productores de Campeche, con los cuales establece varios tipos de relación. La más común son las compras directas ("por la libre”), consistentes en que 
el productor, ya cercana la fecha de cosecha, acuerda con la empresa venderle su soja al precio que esté corriendo en el mercado internacional al momento de la entrega del grano. En menor medida, se realizan contratos de compra-venta muy anteriores a la cosecha (septiembre-octubre), pero éstos, de acuerdo a diversos productores entrevistados, tienen un alto riesgo, ya que se adquiere el compromiso de entregar ciertos volúmenes y calidad que no siempre se pueden cumplir. Es común que tanto Proteínas y Oleicos como Crío (la otra firma compradora) acudan hasta el domicilio de los productores para motivarlos a realizar estos acuerdos contractuales y así asegurarse un cierto abasto de grano. El tercer acuerdo se da en el marco del programa de apoyos del gobierno llamado "Agricultura por Contrato", cuyo principal componente son las coberturas de precios, y que empezó a subsidiar la soja de la Península de Yucatán a partir de 2008 (Conde, 2013, entrevista directa). En dicho programa, productores y compradores del grano firman contratos de compra-venta, en donde se establecen volúmenes, calidad, fechas de entrega, y el "precio de contrato" (precio internacional más bases) que recibirá el productor al momento de la entrega y facturación del grano. Estos contratos son ingresados a ASERCA (Apoyos y Servicios a la Comercialización Agropecuaria), órgano desconcentrado de SAGARPA (Secretaría de Agricultura, Ganadería, Desarrollo Rural, Pesca y Alimentación), quien los revisa, avala y, posteriormente, procede a comprar coberturas de precios en CBOT (Bolsa de Futuros de Chicago), que es donde se fijan los precios internacionales de los granos. Estas coberturas son instrumentos financieros que protegen tanto a productores como a compradores de futuras fluctuaciones de los precios que pudiesen perjudicarles. Al productor le asegura la futura venta de su grano a un precio mínimo (aunque si el precio internacional se incrementa puede acceder a este beneficio), mientras que al comprador le asegura el abastecimiento de grano y lo protege ante futuras alzas de las cotizaciones internacionales (Echanove, 2013). El principal subsidio otorgado por el gobierno es el pago de cierto porcentaje de las "primas" o costo de adquisición de las coberturas. En 2014, el gobierno subsidió con el $100 \%$ de dicho costo a las empresas compradoras de soja (coberturas "put"), las cuales se comprometían a pagar a los productores el precio internacional del grano vigente al momento de su entrega. En 2013, en Campeche, se apoyó con el programa Agricultura por Contrato un total de 18,122 toneladas de soja procedentes de 96 productores (ASERCA, 2014), cifras que representaron el 55\% de la producción estatal, apoyando a casi la mitad de los productores de ese grano.

Y todavía más relevante que el programa de subsidios descrito, por el número de productores beneficiados, ha sido el programa llamado Pro-Oleaginosas, implementado por el gobierno mexicano en 2009 con fines de reconversión productiva. El objetivo era expandir la producción de ese tipo de granos (soja, cártamo, canola, girasol, ajonjolí y cacahuate), en sustitución fundamentalmente del maíz. Durante ese año y 2010 consistió en otorgar a los productores un monto de \$1.100 MXN por hectárea cultivada, lo que se modificó a partir de 2011, en que los apoyos se han venido otorgando en función del volumen de soja vendido a las firmas 
compradoras (aceitera y pecuaria). El apoyo hasta hoy es de \$1.500 MXN por tonelada, existiendo, sin embargo, ciertos límites: solo se subsidia hasta 2 toneladas por hectárea, y hasta un máximo de 200 hectáreas por productor al año, es decir, el monto máximo de apoyo por productor es de 600.000 MXN (34.623 euros). En 2014, Pro-Oleaginosas apoyó a la totalidad de los 200 productores de soja existentes en Campeche (Chi-Bonilla, 2015). Es importante aclarar que éstos agricultores pueden acceder simultáneamente a los beneficios de este programa y a los de Agricultura por Contrato.

Finalmente, otro factor que ha contribuido a la expansión de la soja han sido la eficaz distribución de la semilla GM por parte de Monsanto, la cual, a diferencia de la Huasteca, no ha tenido problemas de oferta o abasto, siendo eficientemente distribuida a través de las llamadas “casas financieras”. Estas conforman la principal fuente de financiamiento de los productores de soja, quienes reciben a crédito la semilla y los insumos químicos, siempre y cuando cuenten con un seguro agrícola. Sólo una minoría de productores obtiene financiamiento de instituciones gubernamentales (Financiera Rural, FIRA) y/o de la banca privada.

\section{Reflexiones finales}

El proceso reseñado en este artículo sobre la expansión de la soja en Campeche constituye un hecho reciente (a partir de 2009) y una estrategia de supervivencia para los productores involucrados que, en su gran mayoría, son menonitas. Sin tener los parámetros cuantitativos de la expansión de esa oleaginosa en otros países de América Latina (Brasil, Argentina, Uruguay y Paraguay) (véase Reboratti, 2010; FAO, 2007), todos ellos comparten ciertas características y efectos. En el caso de Campeche, vimos cómo son varios los elementos que han dado lugar a que la soja sea parte importante del paisaje de ciertos municipios, siendo los más relevantes los siguientes: los subsidios otorgados por el gobierno mexicano en su intento de lograr una reconversión del cultivo del maíz hacia el de soja; la presencia de los menonitas, con el interés y la maquinaria adecuada; la menor demanda de agua de la soja en relación al maíz; los mayores y más estables precios pagados por la soja; el constante abasto de semilla GM por parte de Monsanto; el financiamiento al cultivo por parte de las casas financieras; y el mercado seguro que representa la industria compradora del grano, ubicada en el vecino Estado de Yucatán.

Es innegable que el cultivar soja ha implicado para los productores de Campeche mayores ingresos en relación a otros granos. Sin embargo, la expansión de la soja GM, al igual que en otros países de América Latina, ha tenido efectos negativos. Aunque el estudio de éstos no constituye el objetivo de la presente investigación, señalaremos que entre ellos figura el proceso de deforestación ocurrido sobre todo en Brasil, Argentina, Bolivia y Paraguay (véase WWF, 2014; Reboratti, 2010; Oyhantcabal y Narbondo, 2008). En México, el Estado de Campeche perdió más de 38.000 hectáreas de cobertura forestal en 2013, que en su mayoría fueron cultivadas con soja. En el principal municipio sojero, Hopelchén, donde se encuentra una de las últimas selvas 
de México, tan solo entre 2000 y 2008 se deforestaron 22.300 hectáreas, constituyendo ésta la mayor pérdida de ese país en los últimos años (Regeneración, 2015). Diversas organizaciones civiles han denunciado el papel que en esta destrucción ha jugado el apoyo del gobierno a los productores de soja GM (Navarro, 2015).

Otras repercusiones de la expansión de la soja GM a nivel mundial han sido los daños ocasionados al medio ambiente, en especial la contaminación del agua y la erosión de suelos, como efecto de la aplicación de glifosato, base del herbicida utilizado para el control de malezas en dicho grano (véase Friends of the Earth Europe, 2013). A todo ello, habría que añadir los efectos negativos del mencionado cultivo en la salud humana y animal, así como en la biodiversidad (véase OMS, 2015; GRAIN, 2014; Mason, 2013; Antoniou, 2010; Soy Debate, 2009). La expansión de la soja GM en Campeche, también ha sido materia de conflicto permanente con los productores de miel, quienes han interpuesto y ganado diversos amparos para prohibir dicho cultivo. Los apicultores sostienen que la miel que cosechan contiene polen de las flores de dicha soja, lo que ha afectado sus exportaciones hacia su principal destino, la Unión Europea, en donde los precios de compra se han reducido, además de que el producto no puede ser clasificado como orgánico (Villanueva et al., 2014). En Campeche existen 3.500 productores de miel, cuya producción (8.344 toneladas en 2013) (SDR, 2014) coloca a ese estado entre los principales de México, país que ocupa el cuarto lugar como productor y el quinto como exportador a nivel mundial (STRI, 2014).

La reciente expansión de la soja en Campeche, y en México en general, es también un proceso vulnerable. Su continuidad dependerá del mantenimiento de los apoyos o subsidios del gobierno, sujetos a restricciones presupuestales y factores políticos; a la evolución de los precios internacionales de la soja (y su relación con los del maíz), y al tipo de cambio Peso Mexicano-USD, entre otras variables.

\section{Bibliografía}

Antoniou Michael, Brack Paulo, Carrasco Andrés, Fagan John, Habib, Mohamed, Kageyama Paulo, Leifert Carlo, Onofre Rubens, Pengue, Walter (2010): http://www.gmwatch.org/files/GMsoy_Sust_Respons_SUMMARY_SPA_v1.pdf

ASERCA (Apoyos y Servicios a la Comercialización Agropecuaria) (2014): Dirección Regional Peninsular, Estadísticas del programa de Agricultura por Contrato.

BEbbington, A. (2003): Global networks and local developments: agendas for development geography. Tijdschrift Economische en Sociale Geografie 94 (3): 297-309.

Classen, Abraham (2014): Entrevista realizada por el diario Crónica de Campeche, "Alistan tierras para la siembra de soja", 6 de mayo de 2014.

De hann, L. y ZoomerS, A. (2003): "Development geography at the crossroads of livelihood and globalisation”, Tijdschrift Economische en Sociale Geografie 94 (3): 350-362. 
Delgado, C. (2003): "Rising consumption of meat and milk in developing countries has created a new food revolution”, The Journal of Nutrition 133: 3907S-3910S.

Echanove, F. (2013): Apoyos del gobierno a la comercialización de granos: los programas de agricultura por contrato y coberturas de precios, FUNDAR-Universidad de California Santa Cruz, ANEC-AMUCSS-CCMSs. Pp. 1-37. En: http://subsidiosalcampo.org.mx

FAO (ORGANIZACIÓN PARA LA AGRICULTURA Y LA ALIMENTACIÓN) (2007): Future expansion of soybean: 2005-2014. http://www.fao.org/fileadmin/user_upload/tcas/publications/policy_assistance_series_3.pdf

FAO (ORGANIZACIÓN PARA LA AGRICULTURA Y LA ALIMENTACIÓN) (2016): Soybean Statistics. En: http://faostat3.fao.org/browse/Q/QC/E

Friends of the Earth Europe (2013): "The environmental impacts of glyphosate". http://foeeurope.org/sites/default/files/press_releases/foee_5_environmental_impacts_glyph osate.pdf

GM Soy Debate (2009): "Los impactos agroecológicos de la producción de soja GM en Argentina y Brasil”. En: http://141.105.120.208/dsc/wp-content/uploads/2014/04/Losimpactos-agroecológicos-de-la-producción-de-soja-genéticamente-modificada-enArgentina-y-Brasil.pdf

Grain (2014): “2.4-D soy: waging war on peasants”. En: http://www.grain.org/article/entries/4945-2-4-d-soy-waging-war-on-peasants

Long, N. (2001): Development Sociology. Actor Perspectives. London and New York: Routledge.

Martínez, V. (2014): “Avanza la producción de soja en la región”, Periódico Por Esto, 14 de febrero.

Mason, R. (2013) (comp.): Glyphosate: destructor of human health and biodiversity. En:http://www.uccs.mx/downloads/index.php?id=file_529356c4891e4

Navarro, M. (2015): “Selva de los Chenes en peligro por siembra de soya transgénica”. En: https://elsemanario.com/117986/selva-de-los-chenes-en-peligro-por-siembra-de-soyatransgenica/

OMS (Organización Mundial de la Salud) (2015): "Evaluation of five organophosphate insecticides and herbicides”, IARC Monographs Volume 112. En: http://www.iarc.fr/en/media-centre/iarcnews/pdf/MonographVolume112.pdf

Reboratti, C. (2010): “Un mar de soja: la nueva agricultura en Argentina y sus consecuencias”, Revista de Geografía Norte Grande 45: 63-76.

REGENERACIÓN (2016): "Industria de la soya transgénica destruye la selva de Hopelchén, Campeche”. En:http://regeneracion.mx/industria-soya-transgenica-destruye-la-selvahopelchen-campeche/\#comments

SAGARPA (Secretaría de agricultura, GANADERÍA, Desarrollo Rural, Pesca Y Alimentación) (2015a): Servicio de Información Agroalimentaria y Pesquera. En: http://www.siap.gob.mx

SAGARPA (Secretaría de agricultura, GANADERÍA, Desarrollo Rural, Pesca Y Alimentación) (2015b): Delegación en Campeche, Padrón de productores de soja 2013.

SEGOB (Secretaría de Gobernación) (2013): Primer Informe de Gobierno del Presidente Enrique Peña. En: http://www.presidencia.gob.mx/informe/ 
SDR (Secretaría de Desarrollo Rural) (2014): Estadísticas de producción de miel en Campeche.

Soy Australia LTD (2011): Soybeans in the Australian and Global Market. En: http://www.australianoilseeds.com/_data/assets/pdf_file/0005/8177/Industry__and_Mar ket_Review_2011.pdf

STRI (Smithsonian Tropical Research Institute) (2014): GMO soybean pollen threatens Mexican honey sales. En: https://www.stri.si.edu/sites/strinews/PDFs/STRINews_FEB_14_2014.pdf

Suárez, PABLO (2005): Migración y salud en una comunidad rural del estado de Campeche, Tesis de Maestría, CINVESTAV Mérida. http://www.ecologiahumana.mda.cinvestav.mx/images/egresados/tesis_PabloSuarez.pdf

TRIBUNA (2014a): “Menonitas han devastado más de 5 mil hectáreas en zona chenera”, 1 de agosto. En: http://tribunacampeche.com/

TRIBUNA (2014b): “Menonitas desmontan tierras del ejido Iturbide: Santos Che”, 6 de agosto. En: http://tribunacampeche.com/

USDA (Departamento de Agricultura de Estados Unidos) (2015a): Oil Crops Yearbook. http://usda.mannlib.cornell.edu/MannUsda/viewDocumentInfo.do?documentID=1290

USDA (Departamento de Agricultura de Estados Unidos) (2015b): Bases de datos. En: http://apps.fas.usda.gov/gats/default.aspx

USDA (Departamento de Agricultura de Estados Unidos) (2015c): http://www.ers.usda.gov/topics/crops/soybeans-oil-crops/trade.aspx

USDA (Departamento de Agricultura de Estados Unidos) (2016): World Agricultural Production. En: http://apps.fas.usda.gov/psdonline/circulars/production.pdf

Villanueva, R., Echazarreta, C., Roubik D.W. Y Moguel, Y. B., (2014): “Transgenic soybean pollen (Glycine mas L.) in honey from the Yucatán península, Mexico”, Nature: Scientific Reports 4 (4022).

Wallman, S., And Associates (1982): "Living in South London: Perspectives on Battersea 1871-1981”. Aldershot, Gower, for the London School of Economics and Political Science.

Wise, T. (2011): The impacts of US Agricultural Policies in Mexican Producers. En: http://www.ase.tufts.edu/gdae/Pubs/rp/AgricDumping.pdf

WWF (Organización Mundial de Conservación) (2014): El Crecimiento de la Soja: Impactos y Soluciones, WWF International, Gland, Suiza.

Yúñez, A. (2010): Las políticas públicas dirigidas al sector rural: el carácter de las reformas para el cambio estructural. En Ordorica, M. y Prud'homme, J: Los Grandes Problemas de México. El Colegio de México. 


\section{Entrevistas realizadas}

Aguilar, E. (2014): Ejidatario de Carlos Cano Cruz, entrevista directa, 25 de julio de 2013.

Canul, E. (2014): División de Abastos de Proteínas y Oleicos, entrevista directa, 25 de marzo de 2014.

Chi-Bonilla, J. (2015): Jefe del Distrito de Desarrollo Rural Campeche (002), entrevista directa, 24 de febrero.

Conde, J. (2014): Subdirector de Comercialización de ASERCA Peninsular, entrevista directa, 31 de julio.

Conde, J. (2013): Entrevista directa, 19 de marzo.

De Matteis, B. (2013): Gerente de Agroindustrias de la Comercializadora Mayorista del Golfo (CMG), Productor y Socio de Proteínas y Oleicos, entrevista directa, 26 de julio de 2013.

Escalante, E. (2013): Director Regional Peninsular de ASERCA (Agencia de Servicios a la Comercialización y Desarrollo de Mercados Agropecuarios), entrevista directa, 31 de julio.

Harder, J. (2014): Productor de la colonia Las Flores, entrevista directa, 29 de marzo.

Hernández, I. (2013): Director de Abastos de Proteínas y Oleicos, entrevista directa, 31 de julio.

Hernández, I. (2014): Director de Abastos de Proteínas y Oleicos, entrevista directa, 20 de marzo.

Huiber, C. (2014): Entrevista realizada por el diario Crónica de Campeche, “Alistan tierras para la siembra de soja”, 6 de mayo de 2014.

Oliva, F. (2013): Productor de Los Laureles, entrevista directa, 30 de julio de 2013.

Olvera, G. (2013): Ejidatario de Carlos Cano Cruz, entrevista directa, 25 de julio de 2013.

Pérez, J. (2013): Ejidatario de Carlos Cano Cruz, entrevista directa, 25 de julio de 2013.

Rempel, D. (2014): Productor menonita de la colonia La Nueva Trinidad, entrevista directa, 26 de marzo de 2014.

Tlilayatzi, A. (2013): Ejidatario de Carlos Cano Cruz, entrevista directa, 25 de julio de 2013.

Valencia, F. (2013): Ejidatario de Carlos Cano Cruz, entrevista directa, 25 y 26 de julio de 2013.

Wall, P. (2014): Productor de Nuevo Progreso, entrevista directa, 29 de marzo. 